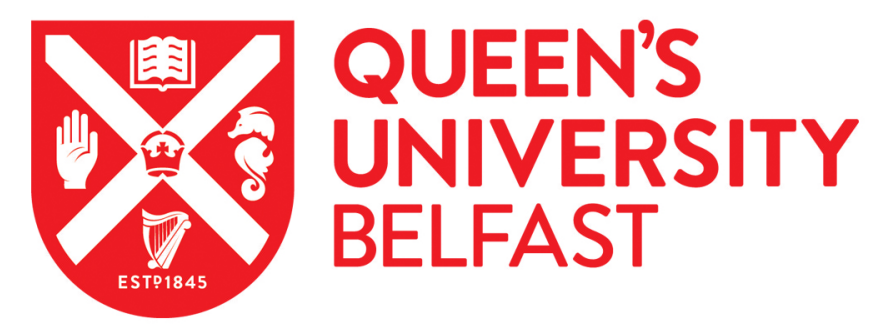

\title{
Robust fault tolerant control for a class second-order nonlinear systems using an adaptive third-order sliding mode control
}

Van, M., Ge, S. S., \& Ren, H. (2017). Robust fault tolerant control for a class second-order nonlinear systems using an adaptive third-order sliding mode control. IEEE Transactions on Systems, Man, and Cybernetics, Part B: Cybernetics. https://doi.org/10.1109/TSMC.2016.2557220

Published in:

IEEE Transactions on Systems, Man, and Cybernetics, Part B: Cybernetics

Document Version:

Peer reviewed version

Queen's University Belfast - Research Portal:

Link to publication record in Queen's University Belfast Research Portal

Publisher rights

Copyright 2017 IEEE. This work is made available online in accordance with the publisher's policies. Please refer to any applicable terms of use of the publisher.

\section{General rights}

Copyright for the publications made accessible via the Queen's University Belfast Research Portal is retained by the author(s) and / or other copyright owners and it is a condition of accessing these publications that users recognise and abide by the legal requirements associated with these rights.

Take down policy

The Research Portal is Queen's institutional repository that provides access to Queen's research output. Every effort has been made to ensure that content in the Research Portal does not infringe any person's rights, or applicable UK laws. If you discover content in the Research Portal that you believe breaches copyright or violates any law, please contact openaccess@qub.ac.uk. 


\title{
Robust Fault Tolerant Control for A Class Second-Order Nonlinear Systems Using an Adaptive Third-order Sliding Mode Control
}

\author{
Mien Van, Shuzi Sam Ge, Fellow, IEEE , Hongliang Ren, Member, IEEE
}

\begin{abstract}
Conventional sliding mode control (SMC) has been extensively developed for design of fault tolerant control (FTC) systems. However, the used of conventional SMC has several disadvantages such as large transient state error, less robustness and large chattering, that limit its application for real application. In order to enhance the performance, a novel passive fault tolerant control (AFTC) based on a chattering-free adaptive third-order sliding mode control (ATOSMC), which integrates a novel third-order sliding mode surface (TOSMS) with a continuous strategy and an adaptation law, is proposed. Compared to other state-of-the-art approaches, the proposed controller has a great fault-tolerant capability to accommodate several types of actuator faults with an enhancing on robustness, precision, chattering reduction and time of convergence. The proposed analytical results are then applied to the attitude control of a spacecraft. Simulation results demonstrate the superior performance of the proposed algorithm.
\end{abstract}

Index Terms-Fault diagnosis, fault tolerant control, highorder sliding mode control, nonlinear systems, sliding mode control.

\section{INTRODUCTION}

$\mathrm{F}$ AULTS, caused by actuators, sensors or other components, frequently occur in practical engineering systems, which might lead to unacceptable performance or in serious cases it might instability and damages or even shut-down of the system [1-2]. In modern technological systems, there is a high demand on performance, safety, and reliability of systems. It is desired that if a fault occurs, the control system can automatically detect the fault and accommodate its effect on the system such that it can continue working while providing an acceptable performance. Therefore, fault tolerant control (FTC) is very important for modern technological systems.

Various FTC approaches have been developed to improve system reliability and to guarantee system stability in all situations [3-13]. In general, FTC systems can be divided into two categories: active and passive approaches [10]. In active approach, FTC is designed based on fault information [3-9, 11-12]. FD is the first step to provide the fault information. The AFTC scheme is then designed based on the obtained fault information in order to compensate for the effect of the fault. The system performance of the AFTC is, therefore, decreased due to the time delay of online fault diagnosis and controller reconfiguration. In passive FTC (PFTC) systems, one controller is used for both the normal case and the fault case without the need to detect the presence of a fault [13-15]. This approach although requires partial knowledge of a possible system fault, it is simple to implement and provide fast response to the effects of faults.

Because the presence of the fault in the system can be considered as an additional uncertainty nonlinear function in the system, intelligent learning techniques such as neural networks (NNs) or fuzzy logic systems (FLSs) have been employed due to its approximation capabilities [5-6, 9, 16-18]. The intelligent learning techniques have the capability to approximate the unknown fault functions in the system; however, they introduce a number of weighting parameters or fuzzy rules that make the implementation difficult. Sliding mode control (SMC) [19] is an efficient control method that has been widely used for both linear and nonlinear system due to its robustness against uncertainties and disturbances [2023]. It has been applied for design of FTC system [3, 24-27]. However, the uses of traditional SMC scheme have several disadvantages that limit its application for FTC. For example, the conventional SMC does not converge to the equilibrium point in finite time because a linear sliding surface is used. Recently, an advance of sliding mode control known as terminal sliding mode control (TSMC) [28], which use nonlinear sliding surface instead of linear surface, have been developed for FTC system $[11,14,29-30]$ to guarantee finite time convergence. Although the aforementioned SMC or TSMC-based FTC robust against the uncertainties and faults, it was reported that the system dynamics might be vulnerable to faults or uncertainties during the reaching phase, where the system states have not yet reached the sliding manifold. To obtain a sliding mode through the entire system response, the advanced of conventional SMC, namely integral sliding mode (ISM), was proposed and further developed in [31-35]. However, all the aforementioned sliding mode controls still have drawbacks such that they produce a large steady state error, slow transient response and the high-frequency oscillations of the controller output, known as chattering. To tackle the first and second obstacles, a new PID-based SMC, in which the PID sliding surface [36-39] or its modified version, called second-order sliding mode surface (SOSMS) [40], have been developed. Compared to the conventional SMC, the PID-based SMC offers several advantages such as 
faster transient response, less tracking error and less steadystate error. To remove the chattering, several solutions have been proposed. One solution is to use the boundary layer method that includes saturation function $[14,23]$ or sigmoid approach [28] instead of sign function. This approach, however, can only guarantee the existence condition of the sliding mode outside a small boundary layer around the sliding manifold, which will increase the steady state tracking errors. Because the chattering depends on the magnitude of the switching gain, that is chosen to be bigger than the bound of the disturbance, a second solution is to design a disturbance observer (DO) to estimate the disturbances [41-42]. After disturbance compensation based on DO, the switching gain only needs to be larger than the bound of the disturbance compensation error, which is usually much smaller than that of the disturbance; consequently, the chattering is reduced. A third solution proposed to decrease the chattering is the highorder sliding mode (HOSM) techniques [43-46]. Unlike the $\mathrm{SMC}$, which works on the first time derivative of the sliding mode variable, HOSM works with discontinuous control acting on the high-order time derivative. By moving the switching to the higher derivatives of the control, the control signal becomes continuous, so the chattering is eliminated.

In this paper, a PFTC based on a novel chattering-free thirdorder sliding mode control (TOSMC), which integrates a third-order sliding mode surface (SOSMS) and a continuous strategy, is first proposed. Compared to other state-of-the-art methods, the proposed control scheme possesses several significant properties that are very important for fault tolerant control system such as strong robustness, higher precision and fast convergence. In addition, like the manner of full-order sliding mode control (FOSMC) [46], the proposed technique based on the TOSMC imposes the system behavior as a desirable full-order dynamics, not a reduced dynamics. However, in the proposed TOSMC approach, the bound of uncertainty, fault and their derivatives must be known in advance for the design of the sliding gains. To overcome this difficult, an adaptive law is employed to adapt the gains, yielding a novel adaptive TOSMC (ATOSMC) is proposed.

The rest of this paper is organized as follows. Section II formulates the problem. The design of the proposed PFTC based on TOSMC and ATOSMC are presented, in section III. The simulation results for tracking control of an attitude control of a spacecraft are given in section IV so as to verify the effectiveness of the proposed algorithms. Finally, the conclusions are given in Section V.

\section{II.PROBLEM STATEMENT}

Consider a class of second-order nonlinear control systems:

$$
\begin{aligned}
& \&_{1}=x_{2} \\
& \&=f(x)+G(x) u+d
\end{aligned}
$$

where $\mathrm{x}_{1}=\left(x_{1}, \ldots, x_{n}\right)^{T} \in \mathfrak{R}^{n}, \mathrm{x}_{2}=\left(x_{n+1}, \ldots, x_{2 n}\right)^{T} \in \mathfrak{R}^{n}$ and $\mathrm{x}=\left(\mathrm{x}_{1}, \mathrm{x}_{2}\right)^{\mathrm{T}} \quad$ represent the system states, $\mathrm{u}=\left(u_{1}, \ldots, u_{m}\right) \in \mathfrak{R}^{m}$ with $m \geq n$ are the control inputs, $\mathrm{d}=\left(d_{1}, \ldots, d_{n}\right)^{T} \in \mathfrak{R}^{n} \quad$ represent the possible model uncertainties and/or disturbances, $\mathrm{f}(\mathrm{x}) \in \mathfrak{R}^{n} \quad$ and $\mathrm{G}(\mathrm{x}) \in \mathfrak{R}^{n \times m}$ are smooth function with $\mathrm{f}(0)=0$, and $(\cdot)^{T}$ denote the transpose of a vector or a matrix.

In this paper, we consider the actuator fault since it often occurs in the nonlinear systems. For the dynamic system (1-2) in the presence of fault, the actuators are divided into two groups $F$ and $H$, within which we assume that during operation, all of the actuators in $H$ must be healthy while those in $F$ are allowed to fail. System (1) becomes:

$$
\begin{aligned}
& \&=x_{2} \\
& \&=f(x)+G_{H}(x) u_{H}+G_{F}(x) u_{F}+d
\end{aligned}
$$

In this paper, we consider the design of $\mathrm{u}_{H}$. Suppose that some or all of the actuators in $F$ work abnormally, (2) becomes [3]:

$$
\begin{aligned}
& \&_{1}=x_{2} \\
& =f(x)+G(x) u+G_{F}(x) \Delta u_{F}+d
\end{aligned}
$$

where $\Delta \mathrm{u}_{F}=\mathrm{u}_{F}^{*}-\mathrm{u}_{F}$, and $\mathrm{u}_{F}^{*}$ and $\mathrm{u}_{F}$ denote the actual and the designed control values for these actuators in $F$.

\section{PFTC BASED ON THIRD-ORDER SLIDING MODE CONTROL}

\section{A. PFTC based on a third-order sliding mode control (TOSMC)}

The basic idea of the proposed PFTC in this paper is to treat the faulty term $\varphi\left(\mathrm{x}, \Delta \mathrm{u}_{F}\right)=\mathrm{G}_{F}(\mathrm{x}) \Delta \mathrm{u}_{F}$ as an additional disturbance and organize a control law based on TOSMC to compensate for the effect of both uncertainty and fault. The design procedure of a TOSMC consists of two main steps. The first step involves constructing the appropriate sliding surface which should have the prescribed desirable dynamical characteristics. The next step is to design a control law that forces the system states to reach the sliding surface in a finite time.

For the first step, the sliding variable is defined and its first and second derivative can be obtained as follows:

$$
\begin{aligned}
& s=x_{1}+\lambda \int x_{1} d t \\
& x_{2}+\lambda x_{1} \\
& x_{2}+\lambda x_{2}
\end{aligned}
$$

where $s=\left(s_{1}, \ldots, s_{n}\right)^{T} \in \mathfrak{R}^{n}$ is the first-order sliding variable, $\lambda>0$ is a design parameter.

Then, the third-order sliding mode surface (TOSMS) is designed as 


$$
\begin{array}{r}
\mathrm{s}_{\mathrm{TOSM}}=a \&+b \mathrm{~s}=2+\lambda \mathrm{x}_{2}+a\left(\mathrm{x}_{2}+\lambda \mathrm{x}_{1}\right) \\
+b\left(\mathrm{x}_{1}+\lambda \int \mathrm{x}_{1} \mathrm{dt}\right) \\
=\mathrm{x}_{2}+(\lambda+a) \mathrm{x}_{2}+(\lambda a+b) \mathrm{x}_{1}+b \lambda \int \mathrm{x}_{1} d t
\end{array}
$$

where $\mathrm{s}_{\text {TOSM }}=\left(s_{1 T O S M}, \ldots, s_{\text {nTOSM }}\right)^{T} \in \mathfrak{R}^{n}$ is third order sliding variable, and $a, b>0$ are design parameters.

The following assumptions are made for the design of FTC based on TOSMC:

Assumption 1: The system uncertainties and faults are satisfied under the following condition:

$\|\varphi(\mathrm{x}, \mathrm{u})+\mathrm{d}\| \leq l_{d}$

where $l_{d}>0$ is a constant.

Assumption 2: There exists a positive constant $k_{d}$ such that

$\left\|\frac{d}{d t}(\varphi(\mathrm{x}, \mathrm{u})+\mathrm{d})\right\| \leq k_{d}$

Based on the assumptions 1 and 2, the design of PFTC based on TOSMC is stated in Theorem 1.

Theorem 1: Consider the nonlinear systems described by (3), if the proposed TOSMC law is designed as (10), in which $\mathrm{u}_{e q}$ is designed as (11) and $\mathrm{u}_{n}$ is designed as (12), then the stability of the proposed TOSMC system can be guaranteed and the tracking error will converge to a neighborhood to zero in finite time.

$$
\begin{aligned}
& \mathrm{u}_{T O S M C}=-\mathrm{G}^{+}(\mathrm{x})\left(\mathrm{u}_{e q}+\mathrm{u}_{n}\right) \\
& \mathrm{u}_{e q}=\left(\mathrm{f}(\mathrm{x})+(\lambda+a) \mathrm{x}_{2}+(\lambda a+b) \mathrm{x}_{1}+b \lambda \int \mathrm{x}_{1} d t\right) \\
& u_{n}+T \mathrm{u}_{n}=v \\
& v=-\left(k_{d}+k_{T}+\eta\right) \operatorname{sign}\left(s_{T O S M}\right)
\end{aligned}
$$

where $\mathrm{G}^{+}(\mathrm{x})=\mathrm{G}^{T}(\mathrm{x})\left[\mathrm{G}(\mathrm{x}) \mathrm{G}^{T}(\mathrm{x})\right]^{-1}, \quad u_{n}(0)=0 ; \quad \eta \quad$ is a positive constant, $k_{d}$ is a constant defined in (9); two constants, $T \geq 0$ and $k_{T}$ are selected to satisfy the following condition:

$k_{T} \geq T l_{d}$

Proof: From system (3), the sliding manifold (7) can be rewritten as follows:

$$
\begin{aligned}
\mathrm{s}_{\mathrm{TOSM}}= & +(\lambda+a) \mathrm{x}_{2}+(\lambda a+b) \mathrm{x}_{1}+b \lambda \int \mathrm{x}_{1} d t \\
=\mathrm{f}(\mathrm{x})+\mathrm{G}(\mathrm{x}) \mathrm{u}+\varphi(\mathrm{x}, \mathrm{u}) & +\mathrm{d}+(\lambda+a) \mathrm{x}_{2} \\
& +(\lambda a+b) \mathrm{x}_{1}+b \lambda \int \mathrm{x}_{1} d t
\end{aligned}
$$

Substituting the control law (10) into above equation gives

$\mathrm{s}_{\mathrm{TOSM}}=\varphi(\mathrm{x}, \mathrm{u})+\mathrm{d}+u_{n}$

The solution of (12) is given by

$$
\begin{aligned}
\mathrm{u}_{n}(t)= & \left(\mathrm{u}_{n}\left(t_{0}\right)+(1 / T)\left(k_{d}+k_{T}+\eta\right) \operatorname{sign}\left(\mathrm{s}_{\mathrm{TOSM}}\right)\right) e^{t-t_{0}} \\
& -(1 / T)\left(k_{d}+k_{T}+\eta\right) \operatorname{sign}\left(\mathrm{s}_{\mathrm{TOSM}}\right)
\end{aligned}
$$

From (14), (16) and (17), the following relationship under the condition $u_{n}(0)=0$ can be obtained:

$k_{T} \geq T l_{d} \geq T\left|u_{n}(t)\right|_{\max } \geq T\left|u_{n}(t)\right|$

i.e., the following inequality will be kept forever:

$T\left|u_{n}(t)\right| \leq k_{T}$

From (16), the derivative of sliding manifold is obtained as

$$
\begin{aligned}
\&_{\mathrm{TOSM}} & =\frac{d}{d t}(\varphi(\mathrm{x}, \mathrm{u})+\mathrm{d})+\iota \& \\
& =\frac{d}{d t}(\varphi(\mathrm{x}, \mathrm{u})+\mathrm{d})+v-T u_{n} \\
& =\frac{d}{d t}(\varphi(\mathrm{x}, \mathrm{u})+\mathrm{d})-\left(k_{d}+k_{T}+\eta\right) \operatorname{sign}\left(s_{T O S M}\right)-T u_{n}
\end{aligned}
$$

Consider the Lyapunov function candidate

$V_{\text {TOSM }}=\frac{1}{2} \mathrm{~s}_{\text {TOSM }}^{T} \mathrm{~s}_{\text {TOSM }}$

Taking derivative of the Lyapunov function and using (20), one can obtain:

$$
\begin{aligned}
& \mathbb{I}_{\text {TOSM }}=\mathrm{s}_{\text {TOSM }}{ }^{T} \& O S M \\
& =\mathrm{s}_{T O S M}{ }^{T}\left(\begin{array}{l}
\frac{d}{d t}(\varphi(\mathrm{x}, \mathrm{u})+\mathrm{d}) \\
-\left(k_{d}+k_{T}+\eta\right) \operatorname{sign}\left(s_{T O S M}\right)-T u_{n}
\end{array}\right) \\
& =\frac{d}{d t}(\varphi(\mathrm{x}, \mathrm{u})+\mathrm{d}) s_{T O S M} \\
& -\left(k_{d}+k_{T}+\eta\right)\left|s_{T O S M}\right|-T u_{n} s_{T O S M} \\
& =\left(\frac{d}{d t}(\varphi(\mathrm{x}, \mathrm{u})+\mathrm{d}) s_{T O S M}-k_{d}\left|s_{T O S M}\right|\right) \\
& +\left(-T u_{n} s_{T O S M}-k_{T}\left|s_{T O S M}\right|\right)-\eta\left|s_{T O S M}\right| \\
& \leq-\eta\left\|\mathrm{s}_{\text {TOSM }}\right\| \\
& <0
\end{aligned}
$$


Therefore, the TOSMC system guarantees the stability of the tracking error, even if parametric uncertainties and external disturbances and faults exist. This completes the proof.

\section{B. PFTC based on adaptive third-order sliding mode control (ATOSMC)}

Although the TOSMC produces less chattering compared to the conventional sliding mode control, the design is based on the restrictive requirements that the assumptions 1 and 2 are guaranteed. It means that the upper bounds of the unknown uncertainty, fault and their derivatives must be known in advance. However, for practical applications, these bound values are difficult to obtain. If the bound is unknown, a large value should be chosen for the sliding gain in order to satisfy the existence condition of the sliding mode (21). Because the controller designed as in (12) and (13) can reduce but not totally eliminate the chattering, the big sliding gain will generate a big chattering, consequently decreasing the system performance. To tackle this challenge, adaptive method is employed into TOSMC, yielding an adaptive TOSMC (ATOSMC).

The overall controller scheme is now designed as

$\mathrm{u}_{\text {ATOSMC }}=-\mathrm{G}^{+}(\mathrm{x})\left(\mathrm{u}_{e q}+\mathrm{u}_{a d}\right)$

where $\mathrm{u}_{e q}$ is designed same as that in (11). $\mathrm{u}_{a d}$ is the adaptive term that is designed as

$\&_{a d}+T \mathrm{u}_{a d}=v_{a}$

$v_{a}=-\left(\hat{K}_{a}+\eta\right) \operatorname{sign}\left(s_{T O S M}\right)$

where the adjustable gain constant $\hat{K}_{a}$ is the estimated value of $\left(k_{d}+k_{T}\right)$, and updated by

$$
\stackrel{\&}{K_{a}}=\frac{1}{\gamma}\left|s_{T O S M}\right|
$$

where $\gamma>0$ denotes the adaptation gain. Choosing a suitable adaptive value $\gamma$ can also effectively avoid high control activity in the reaching mode. The smaller value of adaptive value $\gamma$ will provie a faster convergence system, but generates a bigger value than the desired one.

Define the adaptation error as $\hat{K}_{a}^{0}=\hat{K}_{a}-\left(k_{d}+k_{T}\right)$. Consider a Lyapunov function candidate

$$
V_{\text {ATOSM }}=\frac{1}{2} \mathrm{~s}_{T O S M}{ }^{T} \mathrm{~s}_{T O S M}+\frac{1}{2} \gamma \ddot{K}_{a}^{0}{ }_{K}^{\circ}{ }_{a}^{0}
$$

By taking the derivative of $V_{\text {ATOSM }}$, we obtain

$$
\begin{aligned}
\&_{A T O S M}^{\&}= & \mathrm{s}_{T O S M}{ }^{T}{ }_{T O S M}+\gamma \hat{K}_{a}^{0}{ }^{T} K_{a}^{d} \\
= & \frac{d}{d t}(\varphi(\mathrm{x}, \mathrm{u})+\mathrm{d}) s_{T O S M}-\left(\hat{K}_{a}+\eta\right)\left|s_{T O S M}\right| \\
& \quad-T u_{n} s_{T O S M}+\gamma\left(\hat{K}_{a}-\left(k_{d}+k_{T}\right)\right) K_{a}^{\&}
\end{aligned}
$$

Substituting the adaptation law (25) into (27), it yields

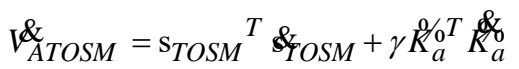

$$
\begin{aligned}
& =\frac{d}{d t}(\varphi(\mathrm{x}, \mathrm{u})+\mathrm{d}) s_{T O S M}-\left(\hat{K}_{a}+\eta\right)\left|s_{T O S M}\right| \\
& -T u_{n} s_{T O S M}+\gamma\left(\hat{K}_{a}-\left(k_{d}+k_{T}\right)\right) \stackrel{\&}{K}_{a} \\
& =\frac{d}{d t}(\varphi(\mathrm{x}, \mathrm{u})+\mathrm{d}) s_{T O S M} \\
& -\left(k_{d}+k_{T}+\eta\right)\left|s_{T O S M}\right|-T u_{n} s_{T O S M} \\
& =\left(\frac{d}{d t}(\varphi(\mathrm{x}, \mathrm{u})+\mathrm{d}) s_{T O S M}-k_{d}\left|s_{T O S M}\right|\right) \\
& +\left(-T u_{n} s_{T O S M}-k_{T}\left|s_{T O S M}\right|\right)-\eta\left|s_{T O S M}\right| \\
& \leq-\eta\left|s_{\text {TOSM }}\right| \\
& <0
\end{aligned}
$$

Using the Lyapunov theorem, the sliding surface $s_{T O S M}$ and adaptation error $\tilde{K}_{a}^{0}$ are stable and convergence. This completes the proof.

Remark 1: To avoid the parameter drift problem in practical applications when using the adaptive law (27), the dead-zone technique is used to modify the adaptive law as

$K_{a}^{\&}= \begin{cases}0 & \text { if }\left|s_{\text {TOSM }}\right| \leq \varepsilon \\ \frac{1}{\gamma}\left|s_{\text {TOSM }}\right| & \text { if }\left|s_{\text {TOSM }}\right|>\varepsilon\end{cases}$

where $\varepsilon>0$ is the size of the dead zone. This value is usually chosen as the desired error the system would be obtained.

\section{C.Accelerometer and velocity estimator}

The developed TOSMC in (12-15) and ATOSMC in (2528) require the measurement of position, velocity and accelerometer. However, in the nonlinear systems defined in (3), only the position measurement or position and velocity measurement is available. Hence, an accelerometer (and velocity estimator) is essential for practical implementation of the controller. Basically, the velocity and acceleration signals can be calculated by backward differentiator (BD) technique, as [47]:

$\&(t)=\frac{x_{1(t)}-x_{1(t-L)}}{L}$ 


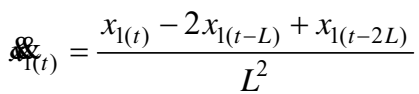

However, it should be noted that the measured position signal contains quantization noise due to the employed encoder [48], which when differentiated would induce significant estimation error. To effectively estimate the velocity and accelerator, second-order exact differentiation (SOED) [45] is introduced in this paper:

\& $=v_{0}$,

$v_{0}=-\xi_{1}\left|z_{0}-x_{1}\right|^{2 / 3} \operatorname{sign}\left(z_{0}-s\right)+z_{1}$

$\&=v_{1}$

$v_{1}=-\xi_{2}\left|z_{1}-v_{0}\right|^{1 / 2} \operatorname{sign}\left(z_{1}-v_{0}\right)+z_{2}$

$\stackrel{\&}{z}=-\xi_{3} \operatorname{sign}\left(z_{2}-v_{1}\right)$

Using suitably chosen parameter $\xi_{i}$, the SOED can achieve

$$
z_{0}=x_{1}, z_{1}=x_{2}, z_{2}=
$$

Remark 2: It should be addressed here that the SOED (32) can achieve finite time error convergence no matter what the kind of controller used. This means that the proposed controller and the observer (32) can be designed separately, so that the combined observer-controller output feedback preserves the main features of the controller with the full state available.

\section{RESULTS AND DISCUSSIONS}

In order to verify the effectiveness of the proposed algorithm, its overall procedure is applied for tracking control of attitude control of a spacecraft.

Consider the following dynamics for spacecraft attitude stabilization control as described in the same form as (1)-(2) with $n=3, \quad$ in which $\mathrm{f}(\mathrm{x})=\left[f_{1}(x), f_{2}(x), f_{3}(x)\right]^{T}$, $\mathrm{x}=\left(\mathrm{x}_{1}, \mathrm{x}_{2}\right)^{\mathrm{T}}, \mathrm{x}_{1}=\left(x_{1}, x_{2}, x_{3}\right)^{T}=(\phi, \theta, \psi)^{T}$,

$\mathrm{x}_{2}=\left(x_{4}, x_{5}, x_{6}\right)^{T}=(\&, \&, 4 \&)^{T}, \mathrm{u}=\left(u_{1}, u_{2}, u_{3}, u_{4}\right)^{T}$, and $\mathrm{d}=\left[d_{1}, d_{2}, d_{3}\right]^{T}$. Here, $\phi, \theta$, and $\psi$ are Euler's angles with respect to $x, y, z$ axes of the spacecraft, respectively, $\mathrm{u}$ denote actuators, providing the force torques by reaction wheels or thrusters in four directions, and $f(x)$ and $G(x)$ components are described by following:

$$
\begin{aligned}
f_{1}(x)= & \omega_{0} x_{6} c x_{3} c x_{2}-\omega_{0} x_{5} s x_{3} s x_{2}+\frac{I_{y}-I_{z}}{I_{x}}\left[x_{5} x_{6}\right. \\
& +\omega_{0} x_{5} c x_{1} s x_{3} s x_{2}+\omega_{0} x_{5} c x_{3} s x_{1}+\omega_{0} x_{6} c x_{3} c x_{1} \\
& +\frac{1}{2} \omega_{0}^{2} s\left(2 x_{3}\right) c^{2} x_{1} s x_{2}+\frac{1}{2} \omega_{0}^{2} c^{2} x_{3} s\left(2 x_{1}\right) \\
& -\omega_{0} x_{6} s x_{3} s x_{2} s x_{1}-\frac{1}{2} \omega_{0}^{2} s^{2} x_{2} s^{2} x_{3} s\left(2 x_{1}\right)
\end{aligned}
$$

\begin{tabular}{|c|c|c|}
\hline Controller & Parameters & Value \\
\hline \multirow{7}{*}{ NFTSMC } & $\sigma_{1}$ & $\operatorname{diag}\{1,1,1\}$ \\
\hline & $\sigma_{2}$ & $\operatorname{diag}\{0.8,1.1,3\}$ \\
\hline & $\alpha$ & 1.4 \\
\hline & $p$ & 9 \\
\hline & $q$ & 7 \\
\hline & $\rho_{d}$ & 20 \\
\hline & $\psi$ & 1000 \\
\hline \multirow{7}{*}{ FOSMC } & $c_{1}$ & 10 \\
\hline & $c_{2}$ & 7 \\
\hline & $\alpha_{1}$ & $9 / 23$ \\
\hline & $\alpha_{2}$ & $9 / 16$ \\
\hline & $T$ & 0.5 \\
\hline & $k_{d}+k_{T}$ & 20 \\
\hline & $\eta$ & 0.5 \\
\hline \multirow{4}{*}{ TOSMC } & $a$ & 10 \\
\hline & $b$ & 5 \\
\hline & $\lambda$ & 10 \\
\hline & $l_{d}+k_{T}, T$ & $20,0.5$ (same as FOSMC) \\
\hline \multirow{2}{*}{ ATOSMC } & $\gamma$ & 0.5 \\
\hline & $\varepsilon$ & 0.01 \\
\hline
\end{tabular}

TABLE I

SELECTED PARAMETERS OF THE CONTROLLERS

$$
\left.-\frac{1}{2} \omega_{0}^{2} s\left(2 x_{2}\right) s x_{2} s^{2}\left(x_{1}\right)-\frac{3}{2} \omega_{0}^{2} c^{2} x_{2} s\left(2 x_{1}\right)\right]
$$

$$
\begin{aligned}
f_{2}(x)= & \omega_{0} x_{6} s x_{3} c x_{1}+\omega_{0} x_{4} c x_{3} s x_{1}+\omega_{0} x_{6} c x_{3} s x_{2} s x_{1} \\
+ & \omega_{0} x_{5} s x_{3} c x_{2} s x_{1}+\omega_{0} x_{4} s x_{3} s x_{2} c x_{1} \\
+ & {\left[\begin{array}{l}
I_{z}-I_{x} \\
I_{y}
\end{array}\left[\begin{array}{l}
x_{4} x_{6}+\omega_{0} x_{4} c x_{1} s x_{3} s x_{2}+\omega_{0} x_{4} c x_{3} s x_{1} \\
-\omega_{0} x_{6} s x_{3} c x_{2}-\frac{1}{2} \omega_{0}^{2} s\left(2 x_{2}\right) s^{2}\left(x_{3}\right) c x_{1} \\
-\frac{1}{2} \omega_{0}^{2} c x_{2} s x_{1} s\left(2 x_{3}\right)+\frac{3}{2} \omega_{0}^{2} s\left(2 x_{2}\right) c x_{1}
\end{array}\right]\right.}
\end{aligned}
$$


$f_{3}(x)=\omega_{0} x_{4} s x_{1} s x_{3} s x_{2}-\omega_{0} x_{6} c x_{1} c x_{3} s x_{2}-\omega_{0} x_{5} c x_{1} s x_{3} c x_{2}$

$+\omega_{0} x_{6} s x_{3} s x_{1}-\omega_{0} x_{4} c x_{3} c x_{1}$

$$
+\frac{I_{x}-I_{y}}{I_{z}}\left[\begin{array}{l}
x_{4} x_{5}+\omega_{0} x_{4} c x_{3} c x_{1}-\omega_{0} x_{4} s x_{3} s x_{2} s x_{1} \\
-\omega_{0} x_{5} s x_{3} c x_{2}-\frac{1}{2} \omega_{0}^{2} s\left(2 x_{3}\right) c x_{2} c x_{1} \\
+\frac{1}{2} \omega_{0}^{2} s^{2} x_{3} s x_{1} s\left(2 x_{2}\right)-\frac{3}{2} \omega_{0}^{2} s\left(2 x_{2}\right) s x_{1}
\end{array}\right]
$$$$
G(x)=\left(\begin{array}{cccc}
0.67 & 0.67 & 0.67 & 0.67 \\
0.69 & -0.69 & -0.69 & 0.69 \\
0.28 & 0.28 & -0.28 & -0.28
\end{array}\right)
$$

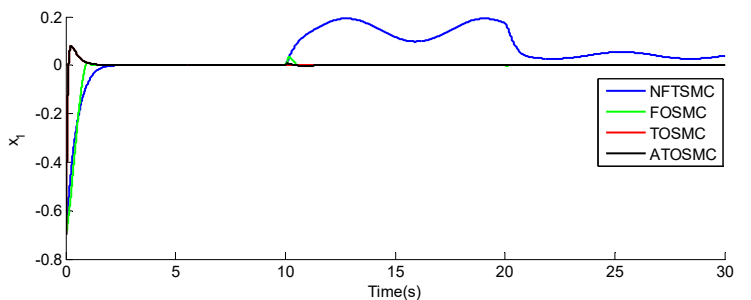

(a)

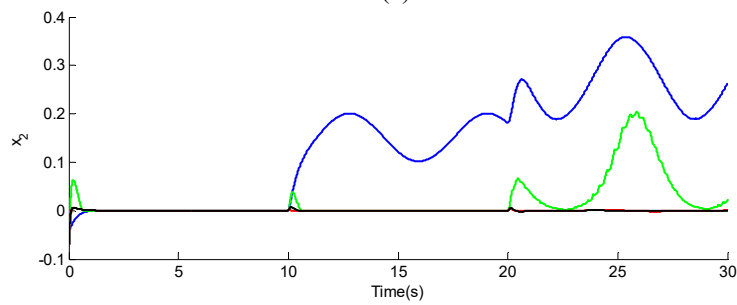

(b)

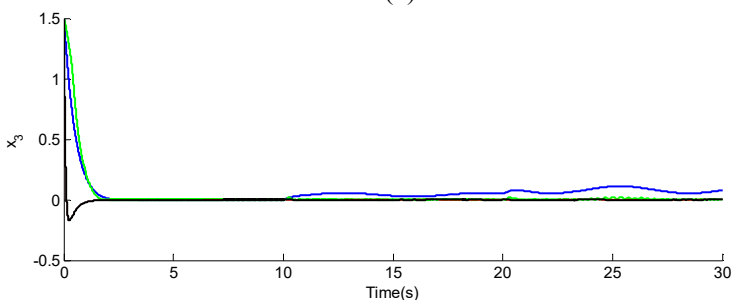

(c)

Fig. 1. Time history of system states $x_{1}, x_{2}, x_{3}$ under the effect of fault $\Delta u_{1}$

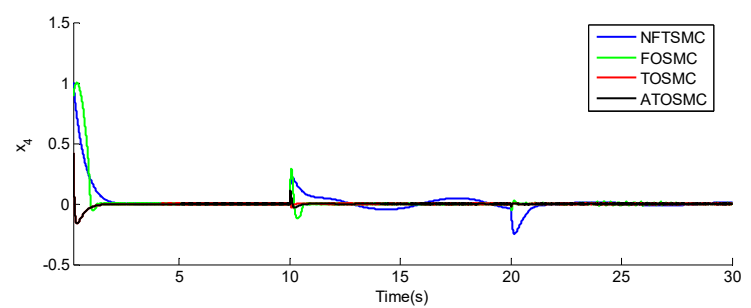

(a)

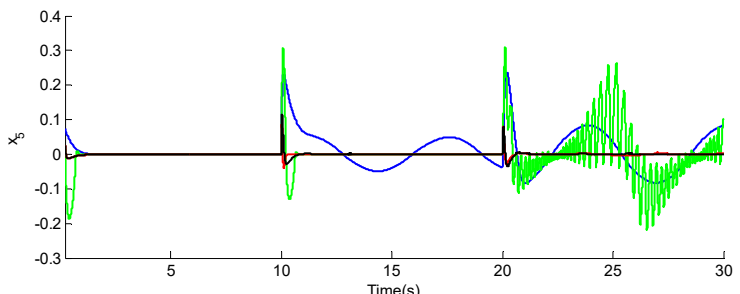

(b)

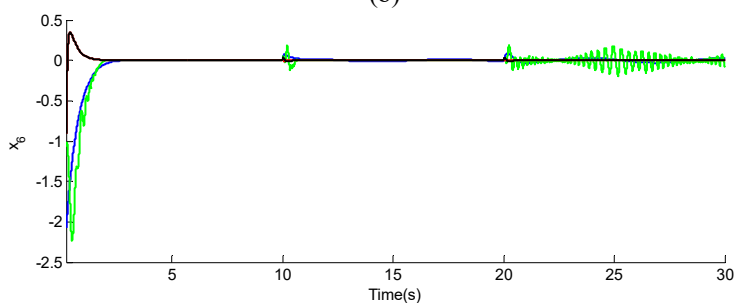

(c)

Fig. 2. Time history of system states $x_{4}, x_{5}, x_{6}$ under the effect of fault $\Delta u_{1}$

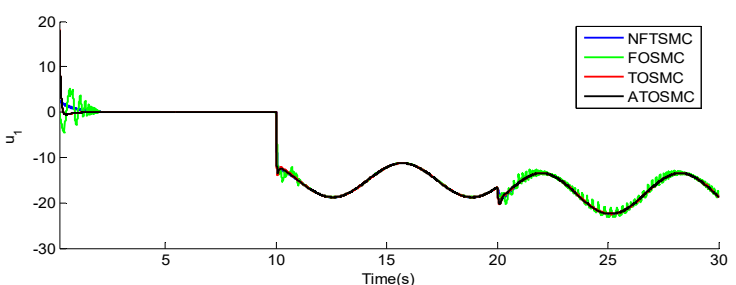

(a)

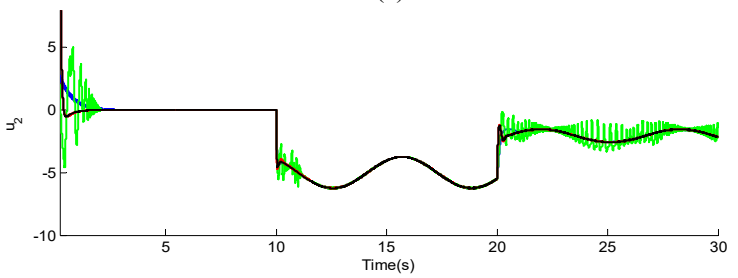

(b)

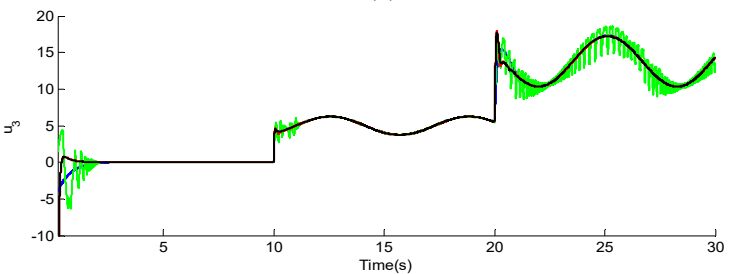

(c)

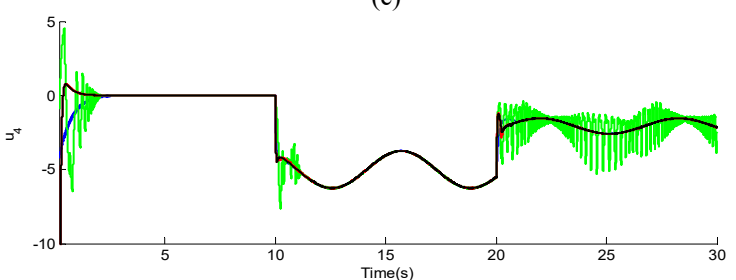

(d)

Fig. 3. Time history of four control inputs 


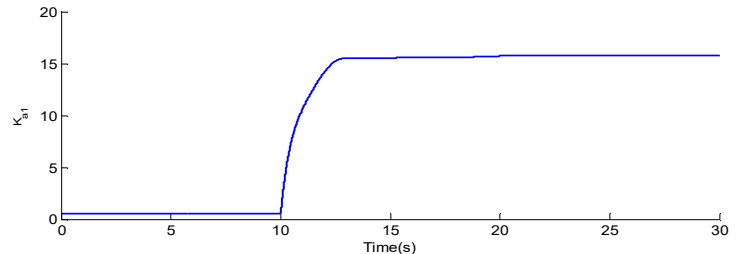

(a)

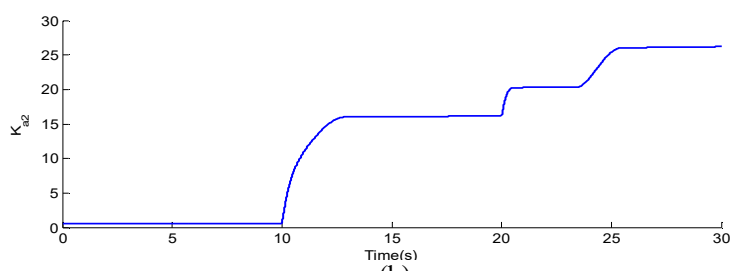

(b)

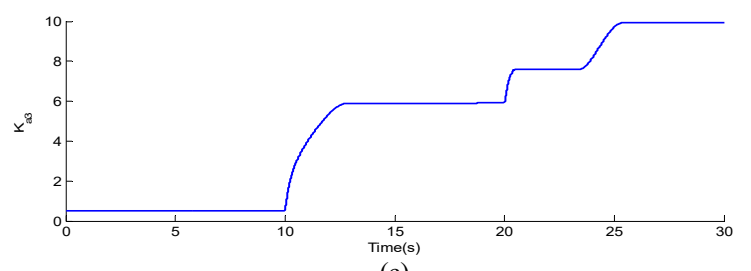

(c)

Fig. 4. Response of the estimated parameters $K_{a 1}, K_{a 2}$ and $K_{a 3}$

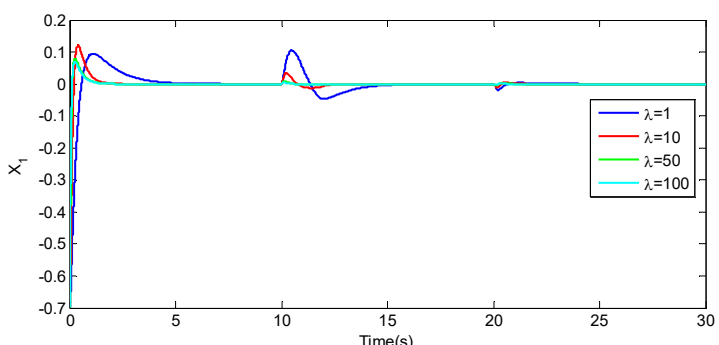

(a)

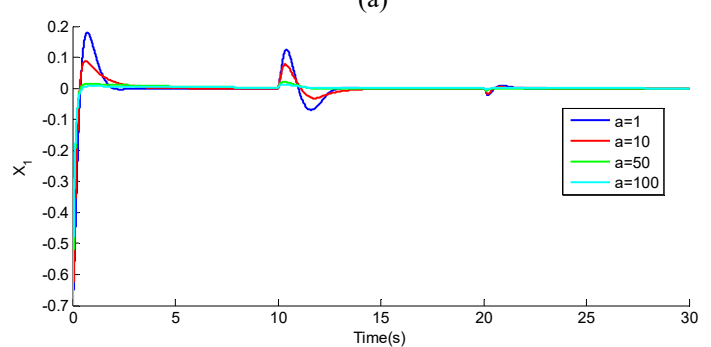

(b)

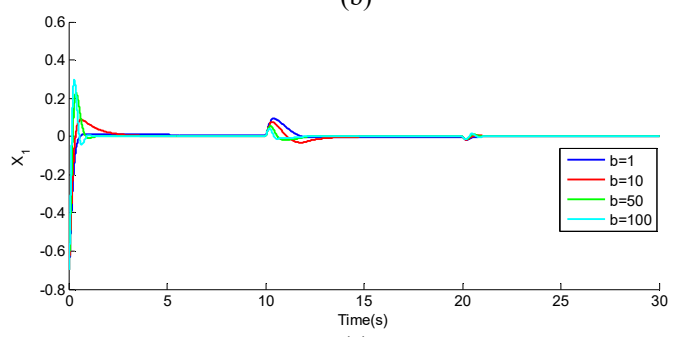

(c)

Fig. 5. Effects of the design parameters, $\lambda, a$ and $b$ on the contro performance. a) $a=10, b=10$ and $\lambda$ : variant, b) $\lambda=10, b=10$ and $a$ : variant, and c) $\lambda=10, a=10$ and $b:$ variant.
Here $I_{x}, I_{y}$, and $I_{z}$ are the inertia with respect to the three body coordinate axes, $\omega_{0}$ denotes the constant orbital rate, and $c$ and $s$ denote the $\cos$ and $\sin$ functions, respectively. Details of these parameters can be referred to [3]. The initial condition and disturbance are set as $x(0)=[-0.7,-0.07,1.5,0.3,1.3,-0.2] \quad$ and $d=0.5[\sin (t), \cos (2 t), \sin (3 t)]$.

In order to verify the effectiveness of the proposed PFTC based on TOSMC and ATOSMC controllers for attitude control of a spacecraft, we compare it with the recent developed sliding mode controllers based on nonsingular fast terminal sliding mode control (NFTSMC) [11] and FOSMC [46]. The design of PFTCs based on NFTSMC and FOSMC are described in the Appendix A and B, respectively. The parameters of the controllers are fairly selected as shown in Table I. The values are selected based on the trial and error evaluation.

In order to evaluate the capability of the controllers in compensating for the effects of faults, we supply an actuator fault $\Delta u=\left[(20+6 \cos (t))(10 s), 0.85 u_{2}(20 s), 0\right]^{T}$; it means we generate in the first actuator a bias fault $\Delta u_{1}=20+6 \cos (t)$ at $10 \mathrm{~s}$, and $85 \%$ partial loss fault in the third actuator at $20 \mathrm{~s}$. The tracking performances of the controllers under the fault $\Delta u$ are shown in Figs. 1-3. Figs. 1 and 2 show the time history of six system states, while Fig. 3 shows the time history of the four control inputs. From Figs. 1 and 2 it can be seen that the proposed TOSMC and ATOSMC controllers have a great fault tolerant capability compared to the NFTSMC and FOSMC controllers, i.e, the TOSMC and ATOSMC provide superior robustness, fast convergence and higher accuracy. The results are coincided with the theoretical analysis and can be explained as follows. Different from the NFTSMC and FOSMC controllers, the sliding surface of the proposed TOSMC imposed the system behavior as a desirable full-order dynamics (like a manner of FOSMC) and contained the type of PID sliding surface as in (4), which would generates faster transient response, less tracking error and less steady-state error as analysis in the introduction part, so that the performance of the proposed TOSMC is better compared to the NFTSMC and FOSMC controllers. In addition, by observing the comparison results in $1 b$ ) and $2 b$ ), the ATOSMC provides better tracking accuracy compared to TOSMC due to the sliding gains are suitable adapted. In addition, from Fig. 3, the TOSMC and ATOSMC provides smooth control efforts compared to the FOSMC. The convergences of the sliding gains of ATOSMC are shown in Fig. 4.

It should be noted that all the design parameters, $\lambda, a$ and $b$ of the proposed sliding surface (7) have a certain effect to the performance of the control system. The big values of $\lambda$, $a$ and $b$ provide faster a convergence but generate a big oscillation. The effects of these parameters on the system performance are illustrated in Fig. 5. 


\section{V.CONCLUSION}

A PFTC based on ATOSMC has been developed for a class of second order nonlinear systems. The structure of the proposed controller is based on the combining of a TOSMS with a continuous strategy and an adaptation law. Compared to other state-of-the-art approaches, the proposed controller has great fault-tolerant capability to accommodate several types of actuator faults with strong robustness, chattering free, fast convergence and does not require prior knowledge of the bound of the uncertainties and faults. The proposed methods are then applied to the attitude control of spacecraft. The results verify the superior performance of the proposed method compared to other state-of-the-art methods.

\section{APPENDIX A}

The PFTC based on NFTSMC for system (3) can be designed as follows [11]:

First, the nonsingular fast terminal sliding mode surface is selected as:

$\mathrm{s}_{N F T S M}=\mathrm{x}_{1}+\sigma_{1} \mathrm{x}_{1}{ }^{[\alpha]}+\sigma_{2} \mathrm{x}_{2}^{[p / q]}$

where $\quad S_{N F T S M} \in \mathfrak{R}^{n} \quad$ is the sliding variable,

$\sigma_{1}=\operatorname{diag}\left(\sigma_{11}, \sigma_{12}, \ldots, \sigma_{1 n}\right) \in \mathfrak{R}^{n \times n}$

and

$\sigma_{2}=\operatorname{diag}\left(\sigma_{21}, \sigma_{22}, \ldots, \sigma_{2 n}\right) \in \mathfrak{R}^{n \times n} \quad$ are positive definite matrices, respectively, $p$ and $q$ are positive odd numbers satisfying the relation $1<p / q<2$ and $\alpha>p / q$, $\mathrm{x}_{1}^{[\alpha]}:=\left(\left|x_{1}\right|^{\alpha} \operatorname{sign}\left(x_{1}\right), \ldots,\left|x_{n}\right|^{\alpha} \operatorname{sign}\left(x_{n}\right)\right)^{T} \in \mathfrak{R}^{n}$, and $\mathrm{x}_{2}{ }^{[p / q]}:=\left(|\& \&|^{p / q} \operatorname{sign}(\&), \ldots,|\&|^{p / q} \operatorname{sign}(\underset{n}{\mathbb{N}})\right)^{T} \in \mathfrak{R}^{n}$.

Under assumption 1 and according to the NFTSMC design procedure [11], a PFTC can be designed as

$u=-\mathrm{G}^{+}(\mathrm{x})\left(u_{e q_{-} N F T S M}+u_{r e_{-} N F T S M}\right)$

where

$$
\begin{aligned}
u_{e q_{-} N F T S M}= & \frac{1}{\sigma_{2}} \frac{q}{p}\left|\mathrm{x}_{2}\right|^{2-(p / q)} \\
& +\sigma_{1} \gamma\left|\mathrm{x}_{1}\right|^{\alpha-1} \frac{1}{\sigma_{2}} \frac{q}{p}\left|\mathrm{x}_{2}\right|^{2-(p / q)}+\mathrm{f}(\mathrm{x})
\end{aligned}
$$

and

$u_{r e \_N F T S M}=\left(\rho_{d}+\eta\right) \operatorname{sign}\left(s_{N F T S M}\right)$

where $\rho_{d}$ is a constant and be selected to satisfy $\varphi(\mathrm{x}, \mathrm{u})+\mathrm{d} \leq \rho_{d}$.

For the stability and convergence, the interested readers can refer to [11] for more detail.
In order to alleviate the chattering phenomenon, the $\operatorname{sign}\left(s_{N F T S M}\right)$ function is replaced by a sigmoid function [27], $\frac{e^{\psi \mathrm{s}_{N F T S M}}-1}{e^{\psi \mathrm{s}_{\text {NFTSM }}}+1}$ in this paper; where $\psi$ is a constant.

\section{APPENDIX B}

The PFTC based on FOSMC for system (5) can be designed as follows [46]:

First, the sliding surface is designed as:

$\mathrm{s}_{F O S M}=c_{2}\left|\mathrm{x}_{2}\right|^{\alpha_{2}} \operatorname{sign}\left(\mathrm{x}_{2}\right)+c_{1}\left|\mathrm{x}_{1}\right|^{\alpha_{1}} \operatorname{sign}\left(\mathrm{x}_{1}\right)$

where $S_{F O S M} \in \mathfrak{R}^{n}$ is the sliding variable, $c_{1}, c_{2}, \alpha_{1}$ and $\alpha_{2}$ are constants.

Based on [45], the PFTC control law can be designed as follows:

$u_{F O S M}=-\mathrm{G}^{+}(\mathrm{x})\left(u_{e q_{-} F O S M}+u_{r_{-} \text {FOSM }}\right)$

where

$u_{e q_{-} F O S M}=\mathrm{f}(\mathrm{x})+c_{2}\left|\mathrm{x}_{2}\right|^{\alpha_{2}} \operatorname{sign}\left(\mathrm{x}_{2}\right)+c_{1}\left|\mathrm{x}_{1}\right|^{\alpha_{1}} \operatorname{sign}\left(\mathrm{x}_{1}\right)$

and

$$
\begin{aligned}
& \&_{\gamma e_{-}} F O S M \\
& \omega=-\left(k_{d}+k_{T}+\eta\right) \operatorname{sign}\left(s_{T O S M}\right)
\end{aligned}
$$

For the stability and convergence, the interested readers can refer to [46] for more detail.

\section{REFERENCES}

[1] H. Noura, D. Theilliol, J. -C. Ponsart, A. Chamseddine, Fault-tolerant control system: design and applications, Springer Verlag, Berlin, Germany, 2009.

[2] M. Blanke, M. Kinnaert, J. Lunze, M. Staroswiecki, Diagnosis andFault-tolerant control system, Springer Verlag, Berlin, Germany, 2003.

[3] Y. W. Liang, S. D. Xu, and C. L. Tsai, "Study of VSC reliable designs with application to spacecraft attitude stabilization," IEEE Trans. Contr. Syst. Technol., vol. 15, no. 2, pp. 332-338, 2007.

[4] C. M. Lin, C. H. Chen, "Robust fault tolerant control for a pibed robot using a recurrent cerebellar model articulation controller," IEEE Trans. Cybenetics., vol.37, no.1, pp. 110-123, 2007.

[5] K. Patan, "Neural network-based model predictive control: fault tolerance and stability," IEEE Trans. Control Syst. Technol., vol. 23, no. 3, pp. 1147-1155, 2015

[6] Q. Shen, B. Jiang, P. Shi, C. C. Lim, "Novel neural networks-based fault tolerant control scheme with fault alarm," IEEE Trans. Cybern., vol. 44, no. 11,2014

[7] Q. Song, W. J. Hu, L. Yin, Y. C. Soh, "Robust adaptive Dead Zone Technology for Fault-Tolerant Control of Robot Manipulators Using Neural Networks," J. Intel. Robot. Syst., vol. 33, no. 2, pp. 113-137, 2002. 
[8] B. Zhao, Y. Li, "Local joint information based active fault tolerant control for reconfigurable manipulator," Nonlinear Dyn., vol. 77, pp 859-876, 2014.

[9] Q. Shen, B. Jiang, V. Cocquempot, "Adaptive fuzzy observer-based active fault-tolerant dynamic surface control for a class of nonlinear systems with actuator faults," IEEE Trans. Fuzzy Syst., vol. 22, no. 2, pp. 338-349, 2014.

[10] Z. Gao, C. Cecati, S. X. Ding, "A survey of fault diagnosis and faulttolerant control techniques Part I: Fault diagnosis with model-based and signal-based approaches," IEEE Trans. Ind. Electron., doi:10.1109/TIE.2015.2417501, 2015.

[11] S. S. D. Xu, C. C. Chen, Z. Lun Wu, "Study of nonsingular fast terminal sliding mode fault-tolerant control," IEEE Trans. Ind. Electron., vol. 62, no. 6, pp. 3906-3913, 2015.

[12] A. Paoli, M. Sartini, S. Lafortune, "Active fault tolerant control of discrete event systems using online diagnostics," IEEE Trans. Vehicular Tech., vol. 60, no. 9, pp. 4276-4287, 2011.

[13] S. M. Tabatabaeipour, R. Izadi-zamanabadi, T. Bak, and A. P. Ravn, "Passive fault tolerant-control of discrete time piecewise affine systems against actuator faults," Int. J. Sys. Sci., vol. 43, no. 11, pp. 1985-1977, 2012.

[14] M. Benosman and K. Y. Lum, "Passive Actuators' Fault tolerant control for affine nonlinear systems," IEEE Trans. Control Syst. Technol., vol. 18 , no. 1 , pp. 152-163, 2010.

[15] R. Wang and J. Wang, "Passive actuator fault-tolerant control for a class of overactuated nonlinear systems and applications to electric vehicles," IEEE Trans. Vehicular Tech., vol. 62, no. 3, pp. 972-985, 2013.

[16] S. Tong and H. X. Li, "Fuzzy adaptive sliding mode control for MIMO nonlinear systems," IEEE Trans. Fuzzy Syst., vol. 11, no. 3, pp. 354-360, 2003.

[17] S. Tong, T. Wang, and Y. Li, "Fuzzy adaptive actuator failure compensation control of uncertain stochastic nonlinear systems with unmodeled dynamics," IEEE Trans. Fuzzy Syst., vol. 22, no. 3, pp. $563-$ 574, 2014.

[18] S. Tong, T. Wang, and Y. Li, "Observer-based adaptive decentralized fuzzy fault tolerant control of nonlinear large-scale systems with actuator failures," IEEE Trans. Fuzzy Syst., vol. 22, no. 1, pp. 1-15, 2014

[19] V. Utkin, Sliding Modes on control and Optimization, Springer Verlag, Berlin, Germany, 1992.

[20] A. Sabanovic, "Variable structure systems with sliding modes in motion control-A survey," IEEE Trans. Ind. Electron., vol. 7, no. 2, pp. 213 223, 2011.

[21] P. Shi, M. Liu, L. Zhang, "Fault tolerant sliding mode observer synthesis of markovian jump systems using quantized measurements," IEEE Trans. Ind. Electro., vol. 62, no. 9, pp. 5910-5918, 2015.

[22] A. Seuret, C. Edwards, S. K. Spurgeon, and E. Fridman, "Static output feedback sliding mode control design via an artificial stabilizing delay," IEEE Trans. Autom. Control, vol. 54, no. 2, pp. 256-265, 2009.

[23] Y. W. Liang, S. D. Xu, and D. C. Liaw, "A study of T-S model-based SMC scheme with application to robot control," IEEE Trans. Ind. Electron., vol. 55, no. 11, pp. 3964-3971, 2008.

[24] H. Alwi and C. Edwards, "Fault tolerant control using sliding modes with online control allocation," Automatica, vol. 44, no. 7, pp. 18591866, Jul. 2008 .

[25] Y. W. Liang and S. D. Xu, "Reliable control of nonlinear systems via variable structure scheme," IEEE Trans. Autom. Control, vol. 51, no. 7, pp. 1859-1866, Jul. 2008.

[26] B. Xiao, Q. L. Hu, and Y. M. Zhang, "Adaptive sliding mode fault tolerant attitude tracking control for flexible spacecraft under actuator saturation," IEEE Trans. Control Syst. Technol., vol. 20, no. 6, pp. 16051612, Nov. 2012.

[27] Q. Hu, B. Xiao, "Fault-tolerant sliding mode attitude control for flexible spacecraft under loss of actuator effectiveness," Nonlinear Dyn., vol. 64 pp. 13-23, 2011.

[28] L. Yang and J. Yang, "Nonsingular fast terminal sliding mode control for nonlinear dynamical systems," Int. J. Robust Nonlinear Control, vol 21, no. 16, pp. 1865-1879, 2011.

[29] J. Li, K. D. Kumar, "Decentralized fault-tolerant control for satellite attitude synchronization," IEEE Trans. Fuzzy Syst., vol. 20, no. 3, pp. 572-586, Jun. 2012.

[30] B. Xiao, Q. Hu, "Finite-time attitude tracking of spacecraft with faulttolerant capability," IEEE Trans. Control Sys. Tech., doi: $\underline{10.1109 / T C S T .2014 .2364124 . ~}$
[31] W. Cao and J. Xu, "Nonlinear integral type sliding surface for both matched and unmatched uncertain systems," IEEE Trans. Autom. Control, vol. 51, no. 5, pp. 1355-1360, Aug. 2004.

[32] F. Castanos and L. Fridman, "Analysis and design of integral sliding manifolds for systems with unmatched perturbations," IEEE Trans. Autom. Control, vol. 51, no. 5, pp. 853-858, May 2006.

[33] M. T. Hamayun, C. Edwards, and H. Alwi, "Design and analysis of an integral sliding mode fault tolerant control scheme," IEEE Trans. Autom. Control, vol. 57, no. 7, pp. 1783-1789, July 2012

[34] Q. Sheng, D. Wang, S. Zhu, E. K. Poh, "Integral-type sliding mode fault tolerant control for attitude stabilization of spacecraft," IEEE Trans. Control Syst. Tech., vol. 23, no. 3, pp. 1131-1138, Sep. 2015.

[35] Y. W. Liang, C. C. Chen, D. C. Liaw, and Y. T. Wei, "Nonlinear reliable control with application to a vehicle antilock brake system," IEEE Trans. Ind. Inform., vol. 9, no. 4, pp. 2114-2123, Nov. 2013.

[36] V. Parra-Vega, S. Arimito, Y.-H. Liu, G. Hirzinger, and P. Akella, "Dynamic sliding PID control for tracking of robot manipulators: Theory and experiments," IEEE Trans. Robot. Automat., vol. 19, no. 6, pp. 967-976, Dec. 2003.

[37] Y. Li, Q. Xu, "Adaptive sliding mode control with pertubation estimation and PID sliding surface for motion tracking of a piezo-driven micromanipulator," IEEE Trans. Control Syst. Tech., vol. 18, no. 4, July 2010.

[38] J. Y. Peng and X. B. Chen, "Integrated PID-based sliding mode state estimation and control for piezoelectric actuators, IEEE/ASM Trans. Mechatron., vol. 19, no. 1, Feb. 2014

[39] Y. Cao and X. B. Chen, "An output tracking based discrete PID-sliding mode control for MIMO systems," IEEE/ASM Trans. Mechatron., vol. 19 , no. 4, 2014

[40] F. J. Lin, Y. C. Hung, K. C. Ruan, “An intelligent second-order sliding mode control for an electric power steering system using a wavelet fuzzy neural network," IEEE Trans. Fuzzy Syst., vol. 22, no. 6, pp. 1598-1611, Jun. 2014

[41] H. Sun, S. Li, C. Sun, "Finite time integral sliding mode control of hypersonic vehicles," Nonlinear Dyn., vol. 73, pp. 229-244, 2013.

[42] Y. Cao and X. B. Chen, "Disturbance-observer-based sliding-mode control for a 3-DOF nanopositioning stage, "IEEE/ASME Trans. Mechatron., vol. 19, no. 3, Jun. 2014.

[43] G. Bartolini, A. Ferrara, E. Usani, "Chattering avoidance by secondorder sliding mode control," IEEE Trans. Autom. Control, vol.43, no.2, pp.241-246, 1998.

[44] Ferrara, M. Rubagotti, "A Sub-Optimal Second Order Sliding Mode Controller for Systems With Saturating Actuators," IEEE Trans. Autom. Control, vol.54, no.5, pp.1082-1087, 2009.

[45] A. Levant, "Robust exact differentiation via sliding mode technique," Automatica, vol. 34, no. 3, pp/ 379-384, 1998.

[46] Y. Feng, F. Han, X. Yu, "Chattering free full-order sliding mode control," Automatica, vol. 50, pp. 1310-1314, 2014.

[47] P. H. Chang, J. W. Jeong, "Enhanced operational space formulation for multiple tasks by using time-delay estimation," IEEE Trans. Robot., vol.28, no.4, pp.773-785, 2012.

[48] J. Zheng and M. Fu, "A reset state estimator using an accelerometer for enhanced motion control with sensor quantization," IEEE Trans. Control Syst. Technol., vol. 18, no. 1, pp. 79-90, 2010. 